\title{
Uptake and cost of influenza vaccines in a private health insured South African population
}

\author{
Geetesh Solanki $^{a, b, c}$, Morna Cornell ${ }^{d, e}$ and Ratilal Lalloo ${ }^{f *}$ (D) \\ ${ }^{a}$ Health System Research Unit, Medical Research Council of South Africa, Cape Town, South Africa \\ ${ }^{b}$ Health Economics Unit, Department of Public Health, University of Cape Town, South Africa \\ ${ }^{c}$ Towers Watson, Cape Town, South Africa \\ ${ }^{d}$ Centre for Infectious Disease Epidemiology \& Research, School of Public Health \& Family Medicine, University of Cape Town, Cape Town, \\ South Africa \\ ${ }^{e}$ Division of Epidemiology and Biostatistics, School of Public Health \& Family Medicine, University of Cape Town, Cape Town, South Africa \\ ${ }^{f}$ School of Dentistry, The University of Queensland, Herston, Queensland, Australia \\ *Corresponding author, email: r.lalloo@uq.edu.au \\ Check for updates
}

Background: Influenza vaccination is regarded as the most effective form of prevention and is particularly recommended for individuals at increased risk of developing severe influenza. Data on uptake (coverage) and costs in South Africa are limited. Methods: This study examined influenza vaccination uptake amongst South African private health insurance scheme members. All claims received for services rendered in 2015 were collated at an individual level and the claims related to the provision of influenza vaccines were analysed.

Results: Of the almost 520000 members, overall 5.0\% (95\% Cl 4.9\%; 5.1\%) received the influenza vaccine in 2015. Priority risk groups such as pregnant women, older adults and those with a medical condition were significantly more likely to be vaccinated, as were members belonging to insurance schemes that offered a specific influenza vaccine benefit. The average cost of providing the vaccination was R350.

Conclusions: Influenza vaccination coverage was low in this privately insured population. There were more members vaccinated in the priority risk groups. There is an urgent need to implement strategies to increase this coverage in privately insured South Africans.

Keywords influenza, private health insurance, South Africa, uptake, vaccination

\section{Introduction}

Seasonal influenza is a highly contagious acute respiratory infection caused by influenza viruses. ${ }^{1,2}$ For most people influenza is an uncomplicated illness lasting three to seven days; however, it can cause serious morbidity and mortality. Worldwide, annual influenza epidemics cause an estimated 3-5 million cases of severe illness and up to 500000 deaths. $^{3}$ As in other temperate countries, annual epidemics occur during autumn and winter in South Africa. The impact of influenza is substantially worse due to the burden of other diseases such as HIV/AIDS, tuberculosis and malaria. In South Africa, influenza represents a serious economic and public health burden, causing an estimated 10000 deaths and 40000 hospitalisations annually. ${ }^{4}$

In Africa, it is argued that influenza is a 'long-overlooked disease', being a major cause of respiratory illness. ${ }^{5,6}$ In 2011 there were almost 50000 cases of severe acute respiratory illness in South Africa. ${ }^{7}$ Of these about $40 \%$ were in HIV-infected individuals, with the majority in children $<5$ years of age. From 1998 to 2009 the mean number of annual child deaths due to influenza was 452 (8 per 100000 person-years) and was significantly

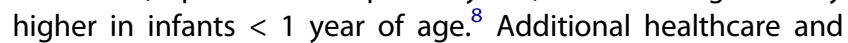
societal impacts of influenza include missed schools and day care amongst children, resulting in missed work days for parents; both non-antibiotic and antibiotic medication; additional burden on healthcare systems; and increased incidence of otitis media. ${ }^{9}$

It is generally believed that influenza viruses are spread by droplets made when people with influenza cough, sneeze or talk. These droplets can infect people by landing in mouths or noses or by being inhaled into the lungs. While general preventative precautions such as hand washing, cough hygiene and avoiding contact with infected individuals can assist in preventing transmission, vaccination is regarded as the most effective form of prevention. Vaccination is particularly recommended for individuals at increased risk of developing severe influenza. ${ }^{10-13}$ A study assessing the risk factors for influenza-associated severe acute respiratory illness requiring hospitalisation found that children $<5$ years of age and older adults $\geq 65$ years of age, as well as those with underlying medical conditions such as HIV, asthma, malnutrition, prematurity, chronic lung and heart diseases and obesity, were at increased risk. ${ }^{13}$ This study also found mineworkers and pregnant women at elevated risk. The vaccination guidelines of the World Health Organization (WHO) prioritise pregnant women, and then, in no specific order, children 6-59 months old, the elderly, individuals with HIV/AIDS, asthma and chronic heart and/or lung diseases, and healthcare workers. ${ }^{3}$ The South African National Influenza Policy and Strategic Plan targets similar high risk-groups in annual vaccination campaigns. ${ }^{4}$

The National Strategic Plan also calls for collaboration with private healthcare providers to ensure broader vaccination coverage. In 2015, approximately 11 million individuals were covered by private health insurance (medical schemes) in South Africa. Most large private health insurance schemes cover the cost of one vaccination per beneficiary per year, although some limit this benefit to individuals in high-risk 
groups. However, little is known about the influenza vaccination uptake and effectiveness in this population. Among 31 African countries, 14 reported availability of the influenza vaccine, and of these only four had vaccine coverage data. ${ }^{14} \mathrm{~A}$ study from the US comparing the influenza vaccination coverage amongst children on the free Vaccine for Children programme and those privately insured showed few differences between the groups. ${ }^{15}$ However, about half of the children did not receive an influenza vaccination in the study period.

The objectives of this paper are to: (1) examine the influenza vaccination uptake amongst South African private health insurance scheme members and assess the extent to which the uptake is in alignment with national guidelines and (2) determine the cost of the vaccine to private health insurance schemes.

\section{Methods}

\section{Data source}

Data were extracted from medical claims records held in the data warehouse of Willis Towers Watson, an independent consulting firm providing consulting and actuarial services to South African private health insurance schemes and pension funds, including 10 schemes covering about a half a million lives ( $\sim 5 \%$ of the privately insured population).

In this study, we analysed claims submitted for reimbursement of healthcare services rendered or items dispensed to members. The following data were extracted: a unique patient identifier; the date on which the service was rendered; a unique identifier for the service provider; a code and description for each service rendered/item dispensed; a National Pharmaceutical Product Index (NAPPI) code for any surgical, medical or consumable item dispensed; and the amount being claimed. The NAPPI code is a unique South African coding system for all pharmaceutical, surgical and healthcare consumable products, which allows providers to claim for these products via a unique funder-recognised code. All claims received from January 1 to December 31, 2015 were collated at an individual level and the claims related to the provision of influenza vaccines were analysed. If the claims for an individual contained any influenza vaccine-related NAPPI codes listed in Table 1, the individual was considered to have been vaccinated for influenza.

Vaccination costs were identified by fees charged for claims against the influenza vaccine-related NAPPI codes listed in Table 1.

\section{Study objectives}

The first objective of the study was assessed by determining vaccination uptake (measured by the proportion of the study population who were immunised in the course of the study period by analytic groups (described later). The second objective of the study was estimated using the costs incurred in covering vaccinations.

\section{Vaccination uptake and costs}

Vaccination uptake per year and vaccination costs were assessed by analytic groups as indicated by the guidelines. Analytic groups included age $(<5$ years, $5-10$ years, $11-20$ years, $21-35$ years, 36-50 years, 51-65 years, 66+ years), gender and maternity status (males, non-pregnant females, pregnant females (females who gave birth at some time in the course of the year), and medical indication for vaccination (indicated if the individual was receiving medication for the treatment of one of the chronic diseases prioritised in the National Strategic
Table 1: List of vaccine products provided to medical scheme members

\begin{tabular}{|l|l|}
\hline NAPPI code & Product description \\
\hline 700767 & Hiberix SGL Dose $0.5 \mathrm{ml}+$ Salin \\
\hline 702733 & X-Flu Prefilled Syringe $0.5 \mathrm{ml}$ \\
\hline 711345 & Fluvax 2009 Prefilled Syringe \\
\hline 711737 & Fluarix Prefilled Syringe $0.5 \mathrm{ml}$ \\
\hline 732826 & Influvac $0.5 \mathrm{ml}$ \\
\hline 732842 & Influvac $5 \mathrm{ml}$ \\
\hline 796441 & Hibtiter 10 MCG \\
\hline 812307 & Agrippal S-1 0.5 $\mathrm{ml}$ Pref S \\
\hline 813257 & X-Flu Single Dose $0.5 \mathrm{ml}$ Inj \\
\hline 813265 & X-Flu M/Dose $5 \mathrm{ml}$ Inj \\
\hline 813338 & Vaxigrip Prefill S/Dose $0.5 \mathrm{ml}$ \\
\hline 813346 & Vaxigrip M/Dose $5 \mathrm{ml}$ \\
\hline 818623 & Fluvirin Prefill \\
\hline 825670 & Vaxigrip 10 Dose Prefill \\
\hline 836591 & Vaxigrip S/Dose 0.25 ml Paed \\
\hline 838853 & Mutagrip Single Dose \\
\hline
\end{tabular}

Plan-see supplemental table for the Chronic Disease List), not indicated if the individual was not being treated for any of the stated conditions. Uptake was also analysed by whether the member belonged to a scheme that offered a specific influenza vaccine benefit (yes or no). South African private health insurance schemes are required by law to cover the costs related to the diagnosis, treatment and care of prescribed minimum benefits (PMBs), to ensure that all members have access to certain minimum health services, regardless of the insurance cover option they have chosen. The PMBs include the Chronic Disease List $(\mathrm{CDL})$, comprising 25 chronic conditions (see supplemental table).

\section{Statistical analysis}

For the vaccination uptake assessment, the vaccination uptake by analytic group (mean percentage with $95 \%$ confidence intervals [Cl]) and the odds ratio with $95 \% \mathrm{Cl}$ for the analytic group versus the reference group are reported. A $95 \% \mathrm{Cl}$ excluding 1 was considered statistically significant. For the cost assessments, the average cost with $95 \% \mathrm{Cl}$ overall and by analytic group were estimated.

Data for the study were made available as part of Willis Towers Watson's commitment to support research initiatives with broader public health significance. The company does not advise its clients on the clinical treatment of members and therefore has no vested interest in the outcome of the study. The data were accessed in terms of and under the conditions set out in the consulting agreement between Willis Towers Watson and their client schemes. The data were analysed by Willis Towers Watson internally and were not made available to any other third party. All findings are presented at an aggregate level and at no point was confidential scheme or member information disclosed.

\section{Results}

\section{Sample description}

The study sample comprised the claims of a total of 519458 individuals over the period from January 1 to December 31, 2015. By age, $7 \%$ of the sample were children less than 5 years and $13.7 \%$ 
were adults older than 65 years of age. The study included slightly fewer males (44.7\%) than females; and of the females, $5.2 \%$ gave birth during the course of the year. By medical condition, $17.3 \%$ suffered from chronic conditions.

\section{Vaccination uptake}

Overall $5 \%(95 \% \mathrm{Cl} 4.9 ; 5.1)$ of the sample were vaccinated for influenza. At $1.6 \%$, vaccination uptake for the $<5$ year age group was the lowest (Table 2). Vaccination uptake was significantly higher for the older ages and highest in the oldest two groups compared with the youngest: $6.1 \%$ for $51-65$ years $(\mathrm{OR}=4.00,95 \% \mathrm{Cl} 3.67 ; 4.36)$ and $18.5 \%$ for $66+$ years $(\mathrm{OR}=$ $13.84,95 \% \mathrm{Cl} 12.73 ; 15.04)$.

Compared with males, non-pregnant females $(\mathrm{OR}=1.12,95 \% \mathrm{Cl}$ $1.09 ; 1.15)$, and pregnant females $(\mathrm{OR}=2.01,95 \% \mathrm{Cl} 1.89 ; 2.13)$ were more likely to be vaccinated. Individuals with medical conditions for which vaccination was indicated were more likely to be vaccinated than those without such indication $(O R=5.55$, $95 \% \mathrm{Cl} 5.41$; 5.69). Similarly, those who had access to a separate vaccine benefit were more likely to be vaccinated than those not having this benefit $(\mathrm{OR}=1.81,95 \% \mathrm{Cl} 1.76 ; 1.87)$.

Table 2: Percentage of medical scheme members vaccinated by analytic group in 2015

\begin{tabular}{|c|c|c|c|}
\hline Factor & $\begin{array}{l}\text { Number of } \\
\text { lives (\%) }\end{array}$ & $\begin{array}{c}\text { Percentage } \\
\text { vaccinated } \\
(95 \% \mathrm{Cl})\end{array}$ & $\begin{array}{c}\text { Odds ratio } \\
(95 \% \mathrm{Cl})\end{array}$ \\
\hline \multicolumn{4}{|l|}{ Age: } \\
\hline$<5$ & $36618(7.0 \%)$ & $1.6(1.5-1.7)$ & 1.00 \\
\hline $5-10$ & 47289 (9.1\%) & $2.0(1.9-2.1)$ & $\begin{array}{c}1.26 \\
(1.14 ; 1.4)\end{array}$ \\
\hline $11-20$ & 59468 (11.4\%) & $1.9(1.8-2.0)$ & $\begin{array}{c}1.18 \\
(1.07 ; 1.3)\end{array}$ \\
\hline $21-35$ & 119359 (23.0\%) & $1.8(1.7-1.9)$ & $\begin{array}{c}1.13 \\
(1.03 ; 1.24)\end{array}$ \\
\hline $36-50$ & 103418 (19.9\%) & $2.9(2.8-3.0)$ & $\begin{array}{c}1.82 \\
(1.66 ; 1.99)\end{array}$ \\
\hline $51-65$ & 82176 (15.8\%) & $6.1(6.0-6.3)$ & $\begin{array}{c}4.00 \\
(3.67 ; 4.36)\end{array}$ \\
\hline $66+$ & 71130 (13.7\%) & $18.5(18.2-18.8)$ & $\begin{array}{c}13.84 \\
(12.73 ; 15.04)\end{array}$ \\
\hline \multicolumn{4}{|c|}{$\begin{array}{l}\text { Gender and } \\
\text { maternity } \\
\text { status: }\end{array}$} \\
\hline Male & 232100 (44.7\%) & $4.6(4.5-4.7)$ & 1.00 \\
\hline $\begin{array}{l}\text { Non- } \\
\text { pregnant } \\
\text { female }\end{array}$ & 272373 (52.4\%) & $5.1(5.1-5.2)$ & $\begin{array}{c}1.12 \\
(1.09 ; 1.15)\end{array}$ \\
\hline $\begin{array}{l}\text { Pregnant } \\
\text { female }\end{array}$ & $14984(5.2 \%)$ & $8.8(8.4-9.3)$ & $\begin{array}{c}2.01 \\
(1.89 ; 2.13)\end{array}$ \\
\hline \multicolumn{4}{|l|}{$\begin{array}{l}\text { Medical } \\
\text { indication? }\end{array}$} \\
\hline No & 429710 (82.7\%) & $3.0(3.0-3.1)$ & 1.00 \\
\hline Yes & 89748 (17.3\%) & $14.6(14.4-14.9)$ & $\begin{array}{c}5.55 \\
(5.41 ; 5.69)\end{array}$ \\
\hline \multicolumn{4}{|l|}{$\begin{array}{l}\text { Separate } \\
\text { influenza } \\
\text { vaccine } \\
\text { benefit? }\end{array}$} \\
\hline No & 171422 (33.0\%) & $3.3(3.2-3.4)$ & 1.00 \\
\hline Yes & $348036(67.0 \%)$ & $5.8(5.8-5.9)$ & $\begin{array}{c}1.81 \\
(1.76 ; 1.87)\end{array}$ \\
\hline Overall & 519458 & $5.0(4.9-5.1)$ & \\
\hline
\end{tabular}

\section{Vaccination costs}

Overall, vaccinations cost ZAR350 $(95 \% \mathrm{Cl} 348 ; 352)$ per individual. The range of vaccination costs is fairly narrow across the analytic categories and is therefore not detailed here.

\section{Discussion}

In this study of over 500000 members of private health insurance, $5 \%$ had received influenza vaccinations over the course of 2015. Vaccination uptake was lowest among those $<5$ years old and highest in those older than 50 years. Women were more likely to have been vaccinated than men, especially if they were pregnant. Vaccination uptake was higher among individuals with specified medical conditions than those without such indication. Individuals who belonged to health insurance schemes that offered an influenza vaccination benefit were more likely to be vaccinated than those who belonged to schemes that did not offer a vaccination benefit.

The overall vaccination uptake of $5 \%$ is low, compared with the US studies of privately insured populations. These studies report vaccination coverage of $25 \%$ in children and $12 \%$ in adults with $52 \%$ coverage in young children 6-24 months old. ${ }^{16}$ In our study less than $2 \%$ of children and young adults (up to 20 years of age) were vaccinated. Of the adults ( $>20$ years of age) $5 \%$ were vaccinated. The overall coverage, and by age group, is similar to previous studies in South Africa. ${ }^{77,18}$ These studies showed a coverage rate below $5 \%$, with increasing rates in adults. Although general uptake was low, uptake in most priority groups was better: $14 \%$ of the members older than 65 years were vaccinated and other priority risk groups such as pregnant women and those with an underlying medical condition were more likely to be vaccinated. While not analysed as a separate age group in our study, no infants $<1$ year were vaccinated, although mortality due to influenza is highest in this age group. ${ }^{13}$ If the vaccination coverage remains low in this population, annual influenza epidemics will remain high with their significant impacts on morbidity and mortality, increased healthcare costs and quality of life of those affected and their carers.

Cost of health care is often a significant driver of health policy and practice, and this applies to both the public and private healthcare sectors. The vaccination cost analysis for this study considered only the medical cost of providing vaccinations. The case for medical schemes covering vaccinations would need to consider the broader social and economic costs such as quality of life, pain and suffering, school absence, loss of income and productivity, unclaimed related healthcare costs (over-the-counter medication) and additional visits to healthcare providers for related problems. Further research is required to understand the broader social and economic impacts of influenza vaccination to establish a rationale for vaccination. A systematic review of the cost-effectiveness of influenza immunisation programmes reported these to be cost-effective, especially in children, pregnant and postpartum women and high-risk groups. ${ }^{19}$

There is a need for private health insurance companies to encourage vaccination for influenza among their membership. Members who had a separate vaccine benefit were almost twice as likely to be vaccinated as individuals whose health insurance did not offer this benefit. Insurance companies should consider expanding this specific benefit in their packages to increase coverage in their membership. Members may not be 
aware of the benefits of the influenza vaccine for their children and may have misconceptions. It is important that stakeholders, including the private health insurance companies, employers and healthcare providers, address concerns and misconceptions regarding the benefits and risks of the influenza vaccine and offer the evidence to their clients for them to make an informed decision whether to vaccinate themselves and their child(ren). ${ }^{20}$ Members of private health insurance schemes and their dependants will likely have regular contact with their general medical practitioner, for a general check-up, a specific problem or continuing care for an existing problem, or antenatal visits; these visits offer opportunities for the influenza vaccine to be offered and provided. This is especially important for the risk priority groups such as the young, the elderly, pregnant women and those with chronic health conditions who would benefit most. A study in South Africa showed that amongst previously vaccinated diabetics advice from the medical practitioner was the main factor encouraging vaccination. ${ }^{21}$ A study of general practices in the United Kingdom reported seven factors that increased vaccine uptake, including having a lead staff member plan the campaign, measuring performance, personal invitations to eligible patients and provision of the vaccine by midwives. ${ }^{22}$

This analysis has a number of limitations. There is considerable year-on-year movement in the underlying membership making trends analysis over a number of years analytically challenging. The movement is due to changes in medical scheme client base and well as movement of members within individual medical schemes. Only services for which claims were submitted were analysed. Services for which no claims were submitted are not captured in the analysis. This could result in under-recording of vaccination uptake. It is, however, unlikely that an insured person would opt to pay for a service covered by the insurance. Vaccination received elsewhere, for example through the public service, other providers such as pharmacies, employers and schools, may not be captured in this analysis. The study assumes accuracy and validity of claims for the vaccination. Critical analyses of the effectiveness, cost-effectiveness, benefit and utility of the influenza vaccine were not possible due to the constraints of the data available.

Vaccination uptake is generally low at $5 \%$, compared with other studies and goals of $70+\%$ for vaccination coverage. While priority risk groups, for example the young and elderly, pregnant women and those with a chronic condition, were more likely to be vaccinated, coverage rates were low even in these groups. Reasons for low uptake of the influenza vaccination, even when paid for the private health insurance, need further research. Further research is also needed to assess the costeffectiveness, benefit and utility of vaccinating against influenza.

Disclosure statement - No potential conflict of interest was reported by the authors.

Funding - This work was supported by the National Institutes of Health (grant number U01Al069924).

Supplemental data - Supplemental data for this article can be accessed http://dx.doi.org/10.1080/23120053.2018.1504532.

\section{References}

1. Gessner BD, Shindo N, Briand S. Seasonal influenza epidemiology in sub-Saharan Africa: a systematic review. Lancet Infect Dis. 2011;11 (3):223-35

2. Rotrosen ET, Neuzil KM. Influenza: a global perspective. Pediatr Clin North Am. 2017;64(4):911-36.

3. World Health Organization. Influenza: vaccine use Geneva: Switzerland: World Health Organization; 2017 [Available from: http://who.int/influenza/vaccines/use/en/.

4. National Department of Health. National influenza policy and strategic plan: 2017 to 2021. Pretoria: South Africa.

5. Katz MA, Schoub BD, Heraud JM, et al. Influenza in Africa: uncovering the epidemiology of a long-overlooked disease. J Infect Dis. 2012;206 (Suppl 1):S1-4.

6. Radin JM, Katz MA, Tempia S, et al. Influenza surveillance in 15 countries in Africa, 2006-2010. J Infect Dis. 2012;206(Suppl 1):S14-21.

7. Murray J, Cohen A, Walaza S, et al. Determining the provincial and national burden of influenza-associated severe acute respiratory illness in South Africa using a rapid assessment methodology. PLoS One. 2015;10(7):e0132078.

8. Tempia S, Walaza S, Cohen AL, et al. Mortality associated with seasonal and pandemic influenza among pregnant and nonpregnant women of childbearing age in a high-HIV-prevalence settingSouth Africa, 1999-2009. Clin Infect Dis. 2015;61(7):1063-70.

9. Ambrose CS, Antonova EN. The healthcare and societal burden associated with influenza in vaccinated and unvaccinated European and Israeli children. Eur J Clin Microbiol Infect Dis. 2014;33(4):569-75.

10. Cohen C, Moyes J, Tempia S, et al. Mortality amongst patients with influenza-associated severe acute respiratory illness, South Africa, 2009-2013. PLOS ONE. 2015;10(3):e0118884.

11. Cohen C, Simonsen L, Kang J-W, et al. Elevated influenza-related excess mortality in South African elderly individuals, 1998-2005. Clin Infect Dis. 2010;51(12):1362-9.

12. Cohen C, Simonsen L, Sample J, et al. Influenza-related mortality among adults aged 25-54 years with AIDS in South Africa and the United States of America. Clin Infect Dis. 2012;55(7):996-1003.

13. Tempia S, Walaza S, Moyes J, et al. Risk factors for influenza-associated severe acute respiratory illness hospitalization in South Africa, 2012-2015. Open Forum Infect Dis. 2017;4(1):ofw262.

14. Duque J, McMorrow ML, Cohen AL. Influenza vaccines and influenza antiviral drugs in Africa: are they available and do guidelines for their use exist? BMC Public Health. 2014;14:41.

15. Srivastav A, Zhai $Y$, Santibanez TA, et al. Influenza vaccination coverage of vaccine for children (VFC)-entitled versus privately insured children, United States, 2011-2013. Vaccine. 2015;33(27):3114-21.

16. Antonova E, Ambrose CS, Kern D, et al. Seasonal influenza vaccination trends from 2007-2011 in privately insured children and adults in the United States. Vaccine. 2014;32(48):6563-8.

17. McAnerney JM, Walaza S, Tempia $S$, et al. Estimating vaccine effectiveness in preventing laboratory-confirmed influenza in outpatient settings in South Africa, 2015. Influenza Other Respir Viruses. 2017;11(2):177-81.

18. Ntshoe GM, McAnerney JM, Tempia S, et al. Influenza epidemiology and vaccine effectiveness among patients with influenza-like illness, viral watch sentinel sites, South Africa, 2005-2009. PLoS One. 2014;9 (4):e94681.

19. Ting EEK, Sander B, Ungar WJ. Systematic review of the cost-effectiveness of influenza immunization programs. Vaccine. 2017;35 (15):1828-43.

20. Paterson $P$, Chantler T, Larson HJ. Reasons for non-vaccination: parental vaccine hesitancy and the childhood influenza vaccination school pilot programme in England. Vaccine. 2017. doi:10.1016/j vaccine.2017.08.016. [Epub ahead of print]

21. Olatunbosun OD, Esterhuizen TM, Wiysonge CS. A cross sectional survey to evaluate knowledge, attitudes and practices regarding seasonal influenza and influenza vaccination among diabetics in Pretoria, South Africa. Vaccine. 2017;35(47):6375-86.

22. Dexter $L$, Teare MD, Dexter $M$, et al. Strategies to increase influenza vaccination rates: outcomes of a nationwide cross-sectional survey of UK general practice. BMJ Open. 2012;2(3):e000851. 